\title{
SOME GENERATING FUNCTIONS FOR LAGUERRE AND HERMITE POLYNOMIALS
}

\author{
FRED BRAFMAN
}

1. Introduction. The Laguerre polynomials $L_{n}{ }^{(\alpha)}(x)$ have the following hypergeometric and Rodrigues representation (3, pp. 188, 189):

$$
\begin{aligned}
& L_{n}^{(\alpha)}(x)=\frac{(1+\alpha)_{n}}{n !}{ }_{1} F_{1}\left[\begin{array}{r}
-n ; \\
1+\alpha ;
\end{array}\right], \\
& L_{n}^{(\alpha)}(x)=\frac{e^{x} x^{-\alpha}}{n !} D_{x}^{n}\left[e^{-x} x^{n+\alpha}\right] .
\end{aligned}
$$

The Hermite polynomials $H_{n}(x)$ have the representations (3, p. 193):

$$
\begin{aligned}
& H_{n}(x)=(2 x)^{n}{ }_{2} F_{0}\left(-\frac{1}{2} n,-\frac{1}{2} n+\frac{1}{2} ;-;-x^{-2}\right), \\
& H_{n}(x)=(-1)^{n} e^{x^{2}} D_{x}^{n} e^{-x^{2}}
\end{aligned}
$$

This note will present several generating functions for the $L_{n}{ }^{(\alpha)}(x)$ and $H_{n}(x)$. The result (5) was recently obtained, by an entirely different method, by Weisner (7). The other following results are believed to be new:

$$
\begin{aligned}
& (1-t)^{-1-\alpha+a}(1-t+v t)^{-a} \exp \left(\frac{-x t}{1-t}\right){ }_{1} F_{1}\left[\begin{array}{c}
a ; \\
1+\alpha ;(1-t)(1-t+v t)
\end{array}\right] \\
& =\sum_{n=0}^{\infty}{ }_{2} F_{1}\left[\begin{array}{c}
-n, a ; \\
1+\alpha ;
\end{array}\right] t^{n} L_{n}{ }^{(\alpha)}(x) \text {; }
\end{aligned}
$$

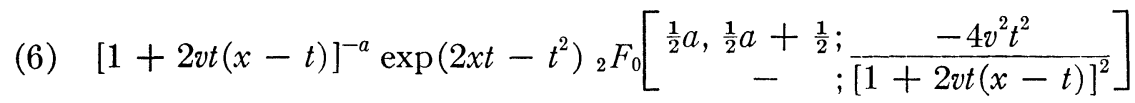

$$
\sim \sum_{n=0}^{\infty}{ }_{2} F_{0}\left[\begin{array}{r}
-n, a ; v \\
-;
\end{array}\right] \frac{t^{n}}{n !} H_{n}(x) ;
$$

(7) $\frac{1}{2} \exp \left(\frac{-x t}{1-t}\right)(1-t)^{a-\alpha-1}\left\{(1-t-v t)^{-a}{ }_{1} F_{1}\left[\begin{array}{c}a ; \\ 1+\alpha ;(1-t)(1-t-v t)\end{array}\right]\right.$

$$
\begin{aligned}
& \left.+(1-t+v t)^{-a}{ }_{1} F_{1}\left[\begin{array}{c}
a ; \\
1+\alpha ;(1-t)(1-t+v t)
\end{array}\right]\right\} \\
& =\sum_{n=0}^{\infty}{ }_{4} F_{3}\left[\begin{array}{cc}
\left.-\frac{1}{2} n,-\frac{1}{2} n+\frac{1}{2}, \frac{1}{2} a, \frac{1}{2} a+\frac{1}{2} ; v^{2}\right] L_{n}{ }^{(\alpha)}(x) t^{n} ; \\
\frac{1}{2}, \frac{1}{2}+\frac{1}{2} \alpha, & 1+\frac{1}{2} \alpha ;
\end{array}\right]
\end{aligned}
$$

Received April 12, 1956. 
(8) $\frac{1}{2}(1-t)^{-1-\alpha} \exp \left(\frac{-x t}{1-t}\right)\left\{\exp \left(\frac{2 v t}{1-t}\right){ }_{0} F_{1}\left[\begin{array}{r}-; \\ \left.1+\alpha ; \frac{-2 v x t}{(1-t)^{2}}\right]\end{array}\right.\right.$

$$
\begin{aligned}
& \left.+\exp \left(\frac{-2 v t}{1-t}\right){ }_{0} F_{1}\left[\begin{array}{r}
-; \\
1+\alpha ; \frac{2 v x t}{(1-t)^{2}}
\end{array}\right]\right\} \\
& =\sum_{n=0}^{\infty}{ }_{2} F_{3}\left[\begin{array}{c}
-\frac{1}{2} n,-\frac{1}{2} n+\frac{1}{2} ; v^{2} \\
\frac{1}{2}, \frac{1}{2}+\frac{1}{2} \alpha, 1+\frac{1}{2} \alpha ;
\end{array}\right] L_{n}^{(\alpha)}(x) t^{n} ;
\end{aligned}
$$

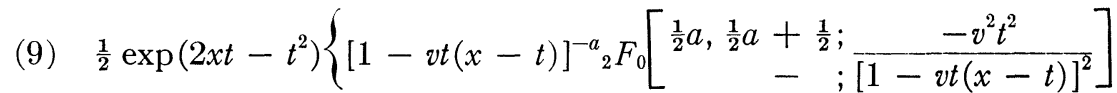

$$
\begin{aligned}
& \left.+[1+v t(x-t)]_{2}^{-a} F_{0}\left[\begin{array}{c}
\left.\frac{1}{2} a, \frac{1}{2} a+\frac{1}{2} ; \frac{-v^{2} t^{2}}{-;}\right] \\
{[1+v t(x-t)]^{2}}
\end{array}\right]\right\} \\
& \sim \sum_{n=0}^{\infty}{ }_{4} F_{1}\left[-\frac{1}{2} n,-\frac{1}{2} n+\frac{1}{2}, \frac{1}{2} a, \frac{1}{2} a+\frac{1}{2} ; v^{2}\right] \frac{H_{n}(x) t^{n}}{n !} \\
& \frac{1}{2} ;
\end{aligned}
$$

(10) $\exp \left[2 x t-t^{2}-v^{2} t^{2}\right] \cosh [2 v t(x-t)]$

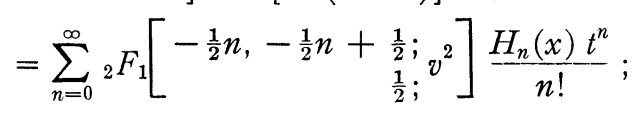

$$
\begin{aligned}
& \exp \left(2 x t-t^{2}\right)\left(1+u t^{2}\right)^{-a}{ }_{1} F_{1}\left[\frac{a ; \frac{u t^{2}(x-t)^{2}}{1+u t^{2}}}{1+2}\right] \\
& =\sum_{n=0}^{\infty}{ }_{3} F_{1}\left[-\frac{1}{2} n,-\frac{1}{2} n+\frac{1}{2}, a ; u\right] \frac{H_{n}(x) t^{n}}{n !} .
\end{aligned}
$$

Equations (6) and (9) give divergent generating functions, but the rest are convergent for $t$ sufficiently small. In equations (5), (6), (7), (9), (11), $a$ is arbitrary. It should be noted that putting $a=-k(k=0,1,2, \ldots)$ in (5) and (7) reduces them to a form involving the general Laguerre polynomial itself, doing this in (11) reduces the left side to a form involving the Laguerre polynomial for the special case $\alpha=-\frac{1}{2}$, and doing this in (6) and (9) reduces the left sides to forms involving the Hermite polynomials. It should further be noted that the

$$
{ }_{2} F_{1}\left[\begin{array}{c}
-\frac{1}{2} n,-\frac{1}{2} n+\frac{1}{2} ; v^{2} \\
\frac{1}{2} ;
\end{array}\right] .
$$

on the right side of (10) are essentially Tchebycheff polynomials of the first kind in the argument $\left(1-v^{2}\right)^{-\frac{1}{2}}$.

2. Proof of (5). From (2) it follows that

$$
L_{n}{ }^{(\alpha)}(x)=\frac{e^{x} x^{-\alpha}}{2 \pi i} \int_{C} \frac{z^{n+\alpha} e^{-z}}{(z-x)^{n+1}} d z,
$$

for $x \neq 0$, where $C$ is a simple closed path about $z=x$, not containing $z=0$. Multiply by

$$
t_{p+1}^{n} F_{q}\left[\begin{array}{r}
-n, c_{1}, c_{2}, \ldots, c_{p} ; \\
d_{1}, d_{2}, \ldots, d_{q} ;
\end{array}\right]
$$


sum over $n$, and switch operations to find:

$$
\begin{aligned}
B(v, x, t) & \equiv \sum_{n=0}^{\infty}{ }_{p+1} F_{q}\left[\begin{array}{r}
\left.-n, c_{1}, c_{2}, \ldots, c_{p} ; v\right] t^{n} L_{n}{ }^{(\alpha)}(x) \\
d_{1}, d_{2}, \ldots, d_{q} ;
\end{array}\right] \\
& \sim \frac{e^{x} x^{-\alpha}}{2 \pi i} \int_{C} \frac{z^{\alpha} e^{-z}}{z-x} A(z) d z,
\end{aligned}
$$

where

$$
A(z)=\sum_{n=0}^{\infty}{ }_{p+1} F_{q}\left[\begin{array}{r}
-n, c_{1}, \ldots, c_{p} ; v \\
d_{1}, \ldots, d_{q} ;
\end{array}\right]\left(\frac{t z}{z-x}\right)^{n} .
$$

Equation (15) can be summed directly or by a special case of a formula of Chaundy (2, p. 62) to give:

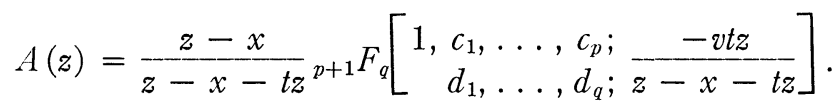

The question of interchange of operations in (14) must now be considered. The preliminary result (18) to be obtained below is general enough to contain both convergent and divergent cases. For $p>q$, the series in (16) diverges and the result is a purely formal one. For $p \leqslant q$, the series converges uniformly for a neighborhood of $t=0$ and the interchange of operations in (14) is justified. In the latter case, replace the equivalence symbol $\sim$ in (14) by equals signs.

In the general case then,

$$
\begin{aligned}
B(v, x, t) & \sim \frac{e^{x} x^{-\alpha}}{2 \pi i} \int_{C} \frac{z^{\alpha} e^{-z}}{z-x-t z}{ }^{p+1} F_{q}\left[\begin{array}{c}
1, c_{1}, \ldots, c_{p} ; \\
d_{1}, \ldots, d_{q} ; z-x-t z
\end{array}\right] d z \\
& \sim \frac{e^{x} x^{-\alpha}}{2 \pi i} \int_{C} \frac{z^{\alpha} e^{-z}}{z-x-t z} \sum_{n=0}^{\infty} \frac{\left(c_{1}\right)_{n} \ldots\left(c_{p}\right)_{n}(-v t z)^{n}}{\left(d_{1}\right)_{n} \ldots\left(d_{q}\right)_{n}(z-x-t z)^{n}} d z \\
& \sim e^{x} x^{-\alpha} \sum_{n=0}^{\infty} \frac{\left(c_{1}\right)_{n} \ldots\left(c_{p}\right)_{n}(-v t)^{n}}{\left(d_{1}\right)_{n} \ldots\left(d_{q}\right)_{n} n !} \frac{n !}{2 \pi i} \int_{C} \frac{z^{\alpha+n} e^{-z}}{(z-x-t z)^{n+1}} d z
\end{aligned}
$$

In the last line, the same comments as to justification of the interchange of operations apply as before. The last line may be converted by (12) provided $t$ is sufficiently small. This gives:

$$
\begin{aligned}
& B(v, x, t) \sim e^{x} x^{-\alpha} \sum_{n=0}^{\infty} \frac{\left(c_{1}\right)_{n} \ldots\left(c_{p}\right)_{n}(-v t)^{n}}{\left(d_{1}\right)_{n} \ldots\left(d_{q}\right)_{n} n !(1-t)^{n+1}}\left[D_{z}^{n}\left(z^{\alpha+n} e^{-z}\right)\right] \\
& \sim \frac{e^{x} x^{-\alpha}}{(1-t)} \sum_{n=0}^{\infty} \frac{\left(c_{1}\right)_{n} \ldots\left(c_{p}\right)_{n}(-v t)^{n}}{\left(d_{1}\right)_{n} \ldots\left(d_{q}\right)_{n}(1-t)^{n} n !}\left[\frac{n ! L_{n}^{(\alpha)}}{e^{z} z^{-\alpha}}\right]_{z=x /(1-t)} \\
& \sim(1-t)^{-1-\alpha} \exp \left(\frac{-x t}{1-t}\right) \sum_{n=0}^{\infty} \frac{\left(c_{1}\right)_{n} \ldots\left(c_{p}\right)_{n}(-v t)^{n}}{\left(d_{1}\right)_{n} \ldots\left(d_{q}\right)_{n}(1-t)^{n}} \\
& L_{n}^{(\alpha)}[x /(1-t)] .
\end{aligned}
$$

Result (5) now follows by taking

$$
p=1, q=1, c_{1}=a, d_{1}=1+\alpha
$$


and using the result (2, p. 62 , quoted in $\mathbf{1}$, p. 949$)$ :

$$
(1-t)^{-a}{ }_{1} F_{1}\left[\begin{array}{cc}
a & ;-x t \\
1+\alpha ; 1-t
\end{array}\right]=\sum_{n=0}^{\infty} \frac{(a)_{n} L_{n}{ }^{(\alpha)}(x) t^{n}}{(1+\alpha)_{n}} .
$$

Another previously known result (6, p. 98, eqn. (5.1.15)) also follows from (18) by taking

$$
p=0, q=1, d_{1}=1+\alpha
$$

and using the result (6, p. 98)

$$
e_{0}^{t} F_{1}(-; 1+\alpha ;-x t)=\sum_{n=0}^{\infty} \frac{L_{n}^{(\alpha)}(x) t^{n}}{(1+\alpha)_{n}} .
$$

3. Proof of (6). The development of (6) follows a pattern similar to that of $\$ 2$. From (4) it follows that

$$
H_{n}(x)=(-1)^{n} \frac{e^{x^{2}} n !}{2 \pi i} \int_{C} \frac{e^{-z^{2}} d z}{(z-x)^{n+1}}
$$

Multiply (23) by

$$
\frac{t^{n}}{n !}{ }^{p+1} F_{q}\left[\begin{array}{r}
-n, c_{1}, \ldots, c_{p} ; \\
d_{1}, \ldots, d_{q} ;
\end{array}\right]
$$

and proceed in a manner exactly like that of $\$ 2$ to find, after two interchanges of operations:

$$
\begin{aligned}
D(v, x, t) & \equiv \sum_{n=0}^{\infty}{ }_{p+1} F_{q}\left[\begin{array}{r}
-n, c_{1}, \ldots, c_{p} ; \\
d_{1}, \ldots, d_{q} ;
\end{array}\right] \frac{t^{n} H_{n}(x)}{n !} \\
& \sim e^{x^{2}} \sum_{n=0}^{\infty} \frac{\left(c_{1}\right)_{n} \ldots\left(c_{n}\right)_{n}(v t)^{n}}{\left(d_{1}\right)_{n} \ldots\left(d_{q}\right)_{n} n !} \frac{n !}{2 \pi i} \int_{C} \frac{e^{-z^{2}} d z}{(z-x+t)^{n+1}} \\
& \sim e^{x^{2}} \sum_{n=0}^{\infty} \frac{\left(c_{1}\right)_{n} \ldots\left(c_{p}\right)_{n}(v t)^{n}}{\left(d_{1}\right)_{n} \ldots\left(d_{q}\right)_{n} n !}\left[(-1)^{n} e^{-z^{2}} H_{n}(z)\right]_{z=x-t} \\
& \sim \exp \left(2 x t-t^{2}\right) \sum_{n=0}^{\infty} \frac{\left(c_{1}\right)_{n} \ldots\left(c_{p}\right)_{n}(-v t)^{n} H_{n}(x-t)}{\left(d_{1}\right)_{n} \ldots\left(d_{q}\right)_{n} n !}
\end{aligned}
$$

Result (6) follows from (25) by taking

$$
p=1, c_{1}=a, q=0,
$$

and using the formula (1, p. 948):

$$
(1-2 x t)_{2}^{-a} F_{0}\left[\begin{array}{c}
\frac{1}{2} a, \frac{1}{2} a+\frac{1}{2} ; \frac{-4 t^{2}}{(1-2 x t)^{2}}
\end{array}\right] \sim \sum_{n=0}^{\infty} \frac{(a)_{n} H_{n}(x) t^{n}}{n !} .
$$

4. Proof of (7). The developments in $\$ \$ 3$ and 4 depended on Chaundy's summation formula, which was used, for example, to convert (15) into (16). The whole procedure may be repeated to yield new results if one will instead use (1, p. 947): 


$$
\begin{aligned}
& (1-t)_{p+2}^{-1} F_{q}\left[\begin{array}{r}
\frac{1}{2}, 1, \alpha_{1}, \ldots, \alpha_{p} ; \frac{x t^{2}}{\beta_{1}, \ldots, \beta_{q} ;} ;(1-t)^{2}
\end{array}\right] \\
& \sim \sum_{n=0}^{\infty} t_{p+2}^{n} F_{q}\left[\begin{array}{r}
-\frac{1}{2} n,-\frac{1}{2} n+\frac{1}{2}, \alpha_{1}, \ldots, a_{p} ; \\
\beta_{1}, \ldots, \beta_{q} ;
\end{array}\right] \text {. }
\end{aligned}
$$

Start again with (12). Then it follows that

$$
\begin{aligned}
E(v, x, t) & \equiv \sum_{n=0}^{\infty}{ }_{p+2} F_{q}\left[\begin{array}{r}
\left.-\frac{1}{2} n,-\frac{1}{2} n+\frac{1}{2}, a_{1}, \ldots, a_{p} ; v^{2}\right] L_{n}^{(\alpha)}(x) t^{n} \\
b_{1}, \ldots, b_{q} ;
\end{array}\right] \\
& \sim \frac{e^{x} x^{-\alpha}}{2 \pi i} \int_{C} \frac{e^{-z} z^{\alpha}}{z-x} A(z) d z
\end{aligned}
$$

where

$$
A(z)=\sum_{n=0}^{\infty}\left(\frac{z t}{z-x}\right)_{p+2}^{n} F_{q}\left[\begin{array}{r}
-\frac{1}{2} n,-\frac{1}{2} n+\frac{1}{2}, a_{1}, \ldots, a_{p} ; v^{2} \\
b_{1}, \ldots, b_{q} ;
\end{array}\right] .
$$

The interchange of operations gives valid convergent results if $p+1 \leqslant q$, and a formal divergent result if $p+1>q$. The application of (28) to (30) and the substitution of the result in (29) yields:

(31) $E(v, x, t)$

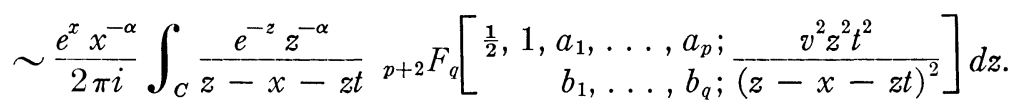

Interchange operations again to find:

(32) $E(v, x, t)$

$$
\sim(1-t)^{-1-\alpha} \exp \left(-\frac{x t}{1-t}\right) \sum_{n=0}^{\infty} \frac{\left(\frac{1}{2}\right)_{n}\left(a_{1}\right)_{n} \ldots\left(a_{p}\right)_{n}(v t)^{2 n}}{\left(b_{1}\right)_{n} \ldots\left(b_{q}\right)_{n}(1-t)^{2 n}} . F
$$

where

$$
F=\frac{x^{-\alpha}(1-t)^{\alpha} \exp [x /(1-t)]}{2 \pi i} \int_{C} \frac{e^{-z} z^{\alpha+2 n} d z}{\left[z-x-\frac{x t}{1-t}\right]^{2 n+1}} .
$$

For $t$ sufficiently small, $x t /(1-t)$ lies within $C$ and hence

$$
F=L_{2 n}{ }^{(\alpha)}[x /(1-t)]
$$

So far then, the result is:

(35) $\quad E(v, x, t)$

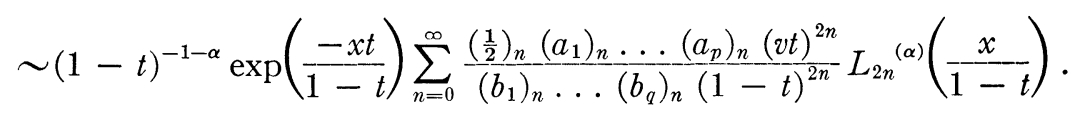

As a special case of (35), take

$$
p=2, q=3, a_{1}=\frac{1}{2} a, a_{2}=\frac{1}{2} a+\frac{1}{2}, b_{1}=\frac{1}{2}, b_{2}=\frac{1}{2}+\frac{1}{2} \alpha, b_{3}=1+\frac{1}{2} \alpha,
$$


and recall that:

$$
(2 c)_{2 n}=2^{2 n}(c)_{n}\left(c+\frac{1}{2}\right)_{n}
$$

Then (35) becomes:

$$
\begin{aligned}
& \sum_{n=0}^{\infty}{ }_{4} F_{3}\left[\begin{array}{r}
\left.-\frac{1}{2} n,-\frac{1}{2} n+\frac{1}{2}, \frac{1}{2} a, \frac{1}{2} a+\frac{1}{2} ; v^{2}\right] L_{n}{ }^{(\alpha)}(x) t^{n} \\
\frac{1}{2}, \frac{1}{2}+\frac{1}{2} \alpha, 1+\frac{1}{2} \alpha:{ }^{2}
\end{array}\right. \\
& \quad=(1-t)^{-1-\alpha} \exp \left(-\frac{x t}{1-t}\right) \sum_{n=0}^{\infty} \frac{(a)_{2 n}(v t)^{2 n}}{(1+\alpha)_{2 n}(1-t)^{2 n} L_{2 n}{ }^{(\alpha)}\left(\frac{x}{1-t}\right) .}
\end{aligned}
$$

From the known result (20), it easily follows that

$$
\begin{aligned}
& (1-t)^{-a}{ }_{1} F_{1}\left[\begin{array}{cc}
a & ;-x t \\
1+\alpha ; 1-t
\end{array}\right]+(1+t)^{-a}{ }_{1} F_{1}\left[\begin{array}{c}
a ; \frac{x t}{1+\alpha ;} \\
1+t
\end{array}\right] \\
& =2 \sum_{n=0}^{\infty} \frac{(a)_{2 n} L_{2 n}{ }^{(\alpha)}(x) t^{2 n}}{(1+\alpha)_{2 n}} .
\end{aligned}
$$

The application of (39) to (38) yields (7).

5. Proof of (8). As a special case of (35), take

$$
p=0, q=3, b_{1}=\frac{1}{2}, b_{2}=\frac{1}{2}+\frac{1}{2} \alpha, b_{3}=1+\frac{1}{2} \alpha .
$$

From the known result

$$
e_{0}^{t} F_{1}(-; 1+\alpha ;-x t)=\sum_{n=0}^{\infty} \frac{t^{n} L_{n}^{(\alpha)}(x)}{(1+\alpha)_{n}},
$$

it is easy to get:

$$
\begin{gathered}
e_{0}^{t} F_{1}(-; 1+\alpha ;-x t)+e^{-t}{ }_{0} F_{1}(-; 1+\alpha ; x t) \\
=2 \sum_{n=0}^{\infty} \frac{t^{2 n} L_{2 n}^{(\alpha)}(x)}{(1+\alpha)_{2 n}} .
\end{gathered}
$$

The application of (42) to the special case represented by (40) gives (8).

6. Proofs of (9), (10), (11). Start from (23), multiply by

$$
\frac{t^{n}}{n !}{ }^{p+2} F_{q}\left[\begin{array}{r}
-\frac{1}{2} n,-\frac{1}{n} n+\frac{1}{2}, a_{1}, \ldots, a_{p} ; v^{2} \\
b_{1}, \ldots, b_{q} ;
\end{array}\right],
$$

sum on $n$, and interchange operations exactly as done previously. The result, stated without intermediate steps is:

$$
\begin{aligned}
& \sum_{n=0}^{\infty}{ }_{p+2} F_{q}\left[\begin{array}{r}
\left.-\frac{1}{2} n,-\frac{1}{2} n+\frac{1}{2}, a_{1}, \ldots, a_{p} ; v^{2}\right] \\
b_{1}, \ldots, b_{q} ;
\end{array}\right] \frac{H_{n}(x) t^{n}}{n !} \\
& \quad \sim \exp \left(2 x t-t^{2}\right) \sum_{n=0}^{\infty} \frac{\left(a_{1}\right)_{n} \ldots\left(a_{p}\right)_{n}\left(\frac{1}{2} v t\right)^{2 n}}{\left(b_{1}\right)_{n} \ldots\left(b_{q}\right)_{n} n !} H_{2 n}(x-t) .
\end{aligned}
$$

In (44), take

$$
p=2, q=1, a_{1}=\frac{1}{2} a, a_{2}=\frac{1}{2} a+\frac{1}{2}, b_{1}=\frac{1}{2} .
$$


From (27), obtain

$$
\begin{aligned}
& (1-2 x t)_{2}^{-a} F_{0}\left[\begin{array}{r}
\frac{1}{2} a, \frac{1}{2} a+\frac{1}{2} ; \frac{-4 t^{2}}{-;(1-2 x t)^{2}}
\end{array}\right] \\
& +(1+2 x t)^{-a}{ }_{2} F_{0}\left[\begin{array}{r}
\left.\frac{1}{2} a, \frac{1}{2} a+\frac{1}{2} ; \frac{-4 t^{2}}{(1+2 x t)^{2}}\right] \\
-;
\end{array}\right. \\
& \sim 2 \sum_{n=0}^{\infty} \frac{(a)_{2 n} H_{2 n}(x) t^{2 n}}{(2 n) !} .
\end{aligned}
$$

The combination of (44), (45), and (46) will yield (9).

As the next special case of (44), take

$$
p=0, q=1, b_{1}=\frac{1}{2} \text {. }
$$

From (6, p. 102)

$$
\exp \left(2 x t-t^{2}\right)=\sum_{n=0}^{\infty} \frac{H_{n}(x) t^{n}}{n !}
$$

obtain :

$$
e^{-t^{2}} \cosh 2 x t=\sum_{n=0}^{\infty} \frac{H_{2 n}(x) t^{2 n}}{(2 n) !} .
$$

The combination (44), (47), and (48) will yield (10).

For the last special case of (44), take

$$
p=1, q=1, a_{1}=a, b_{1}=\frac{1}{2} .
$$

Substitute the result (6, p. 102):

$$
H_{2 n}(y)=(-1)^{n} 2^{2^{n}} n ! L_{n}{ }^{\left(-\frac{1}{2}\right)}\left(y^{2}\right) .
$$

So far, (49) and (50) give, for $v^{2}=u$ :

$$
\begin{aligned}
\sum_{n=0}^{\infty}{ }_{3} F_{1} & {\left[-\frac{1}{2} n,-\frac{1}{2} n+\frac{1}{2}, a ; u\right] \frac{H_{n}(x) t^{n}}{n !} } \\
\frac{\frac{1}{2} ;}{n !} & =\exp \left(2 x t-t^{2}\right) \sum_{n=0}^{\infty} \frac{(a)_{n}\left(-u t^{2}\right)^{n}}{\left(\frac{1}{2}\right)_{n}} L_{n}^{\left(-\frac{1}{2}\right)}\left[(x-t)^{2}\right] .
\end{aligned}
$$

The application of (20) now yields (11).

7. More general results. Make the definition

$$
\begin{aligned}
& f_{n}\left[k ; \begin{array}{c}
\alpha_{1}, \alpha_{2}, \ldots, \alpha_{p} ; x \\
\beta_{1}, \beta_{2}, \ldots, \beta_{q} ;
\end{array}\right] \\
& \quad={ }_{k+p} F_{q}\left[\begin{array}{c}
\frac{-n}{k}, \frac{-n+1}{k}, \ldots, \frac{-n+k-1}{k}, \alpha_{1}, \alpha_{2}, \ldots, \alpha_{p} ; \\
\beta_{1}, \beta_{2}, \ldots, \beta_{q} ;
\end{array}\right],
\end{aligned}
$$

where $k=1,2,3, \ldots$.

Then by direct summation and use of the relation 


$$
(c)_{k j}=k^{k j}(c / k)_{j}((c+1) / k)_{j} \ldots((c+k-1) / k)_{j}
$$

it follows that

$$
\begin{aligned}
& (1-t)^{-a}{ }_{k+p} F_{q}\left[\frac{a}{k}, \frac{a+1}{k}, \ldots, \frac{a+k-1}{k}, \alpha_{1}, \alpha_{2}, \ldots, \alpha_{p} ; x\left(\frac{-t}{1-t}\right)^{k}\right] \\
& \sim \sum_{n=0}^{\infty} \frac{(a)_{n} t^{n}}{n !} f_{n}\left[\begin{array}{l}
\alpha_{1}, \alpha_{2}, \ldots, \alpha_{p} ; x \\
\beta_{1}, \beta_{2}, \ldots, \beta_{q} ; x
\end{array}\right] .
\end{aligned}
$$

Equation (55) is the obvious generalization of (1, p. 947, equs. 24 and 25$)$. For $a=1,(55)$ gives a generalization of (28), which is the special case $k=2$ of $(55)$.

It is thus possible to generalize the procedures of the above sections. The results are so general and so complicated, it does not seem that they would be of much use. Just one of the many possible is presented here for illustration. Let $w_{k}$ be a $k$ th root of unity, other than 1 itself. Then:

$$
\begin{aligned}
& \text { (56) } k^{-1}(1-t)^{-1-\alpha} \exp \left(-\frac{x t}{1-t}\right) \sum_{i=0}^{k-1}\left(1+\frac{w_{k}^{i} t v}{1-t}\right)^{-a} \\
& \sim \sum_{n=0}^{\infty} t^{n} f_{n}\left[\begin{array}{l}
{ }_{1} F_{1}\left[\begin{array}{c}
a \\
1+\alpha ;(1-t)\left(1-t+w_{k}^{i} t v\right)
\end{array}\right] \\
k ; \frac{a}{k}, \frac{a+1}{k}, \ldots, \frac{a+k-1}{k} ; \\
\frac{1}{k}, \frac{2}{k}, \ldots, \frac{k-1}{k}, \frac{1+\alpha}{k}, \frac{2+\alpha}{k}, \ldots, \frac{k+\alpha}{k} ;
\end{array}\right] L_{n}^{(\alpha)}(x) .
\end{aligned}
$$

For $k=2$, (56) reduces to $(7)$ and for $k=1,(56)$ reduces to (5). The interested reader will be able to find similar generalizations of (6) through (11).

\section{REFERENCES}

1. F. Brafman, Generating functions of Jacobi and related polynomials, Proc. Amer. Math. Soc. 2 (1951), 942-949.

2. T. W. Chaundy, An extension of the hypergeometric function, Quart. J. Math., 14 (1943), 55-78.

3. A. Erdélyi, W. Magnus, F. Oberhettinger, F. Tricomi, Higher Transcendental Functions, 2 (New York, 1953).

4. - Higher Transcendental Functions, 3 (New York, 1955).

5. W. Magnus and F. Oberhettinger, Special Functions of Mathematical Physics (New York, 1949).

6. G. Szegö, Orthogonal Polynomials, Colloquium Publications XXIII (Amer. Math. Soc., 1939).

7. L. Weisner, Group-theoretic origin of certain generating functions, Pacific J. Math. 4, supp. 2 (1955), 1033-1039.

Wayne State University and

Southern Illinois University 